\title{
DIRECIONALIDADE E RECONHECIMENTO DE FALA NO RUÍDO: ESTUDO DE QUATRO CASOS
}

\section{Directionality and speech recognition in noise: study of four cases}

\author{
Mariane Turella Mazzochi ${ }^{(1)}$, Aline Domingues Chaves Aita ${ }^{(2)}$
}

\begin{abstract}
RESUMO
A dificuldade de compreensão de fala no ruído é apontada como uma das principais incapacidades pelo usuário de Aparelho de Amplificação Sonora Individual. O objetivo desse estudo foi comparar o desempenho auditivo de sujeitos portadores de perda auditiva neurossensorial, bilateral, de grau leve a moderado, com os microfones ominidirecional, direcional fixo e direcional adaptativo automaticamente ativado, por meio da relação sinal/ruído (S/R) nas quais são obtidos os Limiares de Reconhecimento de Sentenças no Ruído (LRSR). Utilizaram-se os aparelhos Reach, modelo RCH62, da marca Beltone, nos modos microfone omnidirecional, direcional fixo e direcional adaptativo automaticamente ativado. Foram testadas as seguintes situações de apresentação dos estímulos acústicos: fala $0^{\circ}$ azimute e ruído $180^{\circ}$ azimute $\left(0^{\circ} / 180^{\circ}\right)$, fala $90^{\circ}$ azimute e ruído $270^{\circ}\left(90^{\circ} / 270^{\circ}\right)$ azimute e fala $270^{\circ}$ azimute e ruído $90^{\circ}$ azimute $\left(270^{\circ} / 90^{\circ}\right)$. A média das relações sinal/ruído variou de 6,6 $\mathrm{dB}$ a $-6,9 \mathrm{~dB}$. O microfone que apresentou melhor média para a relação sinal/ruído, considerando as três situações de apresentação dos estímulos, foi o direcional adaptativo automaticamente ativado. Entretanto, por se tratar de uma amostra pequena, houve grande variabilidade individual. Mais estudos devem ser realizados a fim de que se tenham subsídios científicos para a seleção dos microfones mais apropriados.
\end{abstract}

DESCRITORES: Auxiliares de Audição; Perda Auditiva; Inteligibilidade da Fala; Ruído

\section{INTRODUÇÃO}

O reconhecimento da fala depende da integridade do sistema auditivo periférico e central e da combinação de pistas como a intensidade, a duração do sinal acústico, a faixa de frequência dos fonemas, a prosódia, a familiaridade com o vocabulário e o contexto linguístico ${ }^{1}$. Em situações de escuta favoráveis o ouvinte consegue descartar algumas dessas pistas sem perder o conteúdo da mensagem.

No entanto, no cotidiano, não é raro deparar-se com situações de escuta desfavoráveis, tais como a presença de fala e ruído ao mesmo tempo. Essa

(1) Acadêmica do Curso de Fonoaudiologia da Faculdade Nossa Senhora de Fátima.

(2) Fonoaudióloga; Professora titular do Curso de Fonoaudiologia da Faculdade Nossa Senhora de Fátima, Caxias do Sul, Rio Grande do Sul, Brasil; Doutor em Ciências dos Distúrbios da Comunicação Humana pela Universidade Federal de São Paulo - UNIFESP.

Conflito de interesses: inexistente condição exige do ouvinte a utilização de todas as pistas disponíveis para que haja compreensão da mensagem.

Para o indivíduo com perda auditiva, mesmo que mínima, todas as pistas advindas do ambiente tornam-se fundamentais para o reconhecimento da mensagem devido ao déficit existente no sistema auditivo. Assim, nas situações de escuta desfavoráveis, o acesso a essas pistas é prejudicado. Portanto, para esses indivíduos, a dificuldade de compreensão da fala em ambientes ruidosos é apontada como uma das principais incapacidades auditivas, pois afeta significantemente sua qualidade de vida, levando muitas vezes ao isolamento social, familiar e profissional.

Uma das maneiras de minimizar esses efeitos tem sido a adaptação do Aparelho de Amplificação Sonora Individual (AASI). O AASI consiste em um sistema que amplifica os sons do ambiente de forma que esses possam ser percebidos pelo usuário. Os AASI'S digitais captam o sinal sonoro do ambiente transformando-o em sinal elétrico equivalente e depois em um padrão digital, modificam-no 
conforme as necessidades e entregam-no ao indivíduo na forma de sinal acústico ${ }^{2}$.

Um AASI é composto basicamente por um microfone, um amplificador e um receptor. O microfone é o componente responsável por converter o sinal acústico do ambiente em sinal elétrico equivalente, sendo assim o transdutor de entrada do AASI. Esse componente pode variar quanto à sensibilidade à direção da fonte sonora, sendo dividindo em dois grandes grupos: omnidirecionais e direcionais ${ }^{2}$.

Os microfones omnidirecionais são os mais usados e captam da mesma maneira os sons vindos de todas as direções. Já os microfones direcionais captam melhor os sons provenientes da frente, reduzindo a sensibilidade aos sons vindo de trás e dos lados ${ }^{2}$.

Atualmente, com o grande avanço da tecnologia, os microfones direcionais podem ser subdivididos em direcionais fixos, direcionais fixos automaticamente ativados, direcionais adaptativos manuais e direcionais adaptativos automaticamente ativados ${ }^{3}$.

Os sistemas de microfones direcionais fixos fornecem um padrão de resposta estático que foca a direcionalidade para frente do indivíduo. Essa estratégia baseia-se na suposição de que o falante estará de frente para o ouvinte e o ruído proverá dos lados ou de trás, entretanto, alguns estudos apontam que o sinal de fala não provém da frente do ouvinte em mais de $20 \%$ das situações ${ }^{4}$.

O microfone direcional adaptativo automaticamente ativado tem por característica principal o acionamento da direcionalidade conforme a informação dada pelo ambiente ${ }^{3}$. Desse modo, o padrão polar varia de acordo com detecção dos sons de fala e de ruído, nível geral do som de entrada e direção dos sinais de fala.

Assim, para distinguir os sinais de fala do ruído, tal sistema parte do princípio de que o último é um som relativamente constante enquanto que a fala varia em intensidade, frequência e duração. Assim que o sinal de fala é identificado, o padrão polar é modificado de forma que esse seja mais amplificado em relação aos demais ${ }^{5}$.

Considerando que a dificuldade de compreender a fala em ambientes ruidosos possa ser um fator limitante para a satisfação do usuário de AASI e que a direcionalidade dos microfones pode contribuir significantemente para a compreensão da fala no ruído, o objetivo desse estudo foi comparar o desempenho auditivo de sujeitos portadores de perda auditiva neurossensorial, bilateral, de grau leve a moderado, com os microfones ominidirecional, direcional fixo e direcional adaptativo automaticamente ativado, por meio da relação sinal/ ruído (S/R) nas quais são obtidos os Limiares de Reconhecimento de Sentenças no Ruído (LRSR).

\section{APRESENTAÇÃO DOS CASOS}

A casuística foi constituída por quatro indivíduos, sendo dois do sexo masculino e dois do sexo feminino na faixa etária entre 56 e 63 anos, sendo a média de idade de 59,8 anos $\pm 3,3$ desvios-padrão, atendidos no Setor de Prótese Auditiva do Centro de Saúde Auditiva Clélia Spinato Manfro, da Faculdade Nossa Senhora de Fátima, no momento da seleção do AASI.

Os pacientes foram selecionados com base na análise de prontuários da lista de espera para o teste de AASI do referido serviço, visando atender aos seguintes critérios de inclusão: ser adulto até 65 anos de idade; portador de perda auditiva neurossensorial de grau leve a moderado (26 a 70dB), conforme classificação proposta por Davis e Silverman, $1970{ }^{6}$, simétrica; estar na lista de espera para submissão ao teste para a seleção da prótese auditiva, no Centro de Saúde Auditiva Clélia Spinato Manfro com indicação para adaptação de AASI binaural. Nenhum deles havia feito uso de ASSI até o momento.

Indivíduo 1: sexo masculino, 62 anos, aposentado. Portador de perda auditiva neurossensorial de grau moderado com configuração descendente em ambas as orelhas. Referiu dificuldade de compreensão de fala tanto em ambientes silenciosos quanto em ambientes ruidosos.

Indivíduo 2: sexo feminino, 56 anos, do lar. Portadora de perda auditiva neurossensorial de grau moderado em ambas as orelhas. Referiu dificuldade de compreensão de fala apenas em ambientes ruidosos.

Indivíduo 3: sexo masculino, 63 anos, encanador e eletricista. Portador de perda auditiva neurossensorial de grau leve em ambas as orelhas. Referiu dificuldade de compreensão de fala apenas em ambientes ruidosos.

Indivíduo 4: sexo feminino, 58 anos, do lar, portadora de perda auditiva neurossensorial de grau leve em ambas as orelhas. Referiu dificuldade de compreensão de fala em algumas situações, tanto em ambientes silenciosos, quanto em ambientes ruidosos.

Primeiramente, os indivíduos foram submetidos a inspeção visual do meato acústico externo. Num segundo, momento realizaram-se os procedimentos padrão para seleção de AASI preconizados pelo serviço.

Na sequência, os quatro sujeitos foram submetidos ao Teste de Reconhecimento de Sentenças no Ruído, proposto por Costa, em 1998, utilizando o aparelho Reach, modelo RCH62, da marca Beltone, adaptação aberta, nas duas orelhas. Por 
fim, responderam ao questionário de avaliação qualitativa elaborado para essa pesquisa.

$O$ teste foi realizado com os microfones dos aparelhos nos modos omnidirecional, direcional fixo cardióide e direcional adaptativo automaticamente ativado. Selecionou-se o raio grande na programação do microfone direcional fixo e raio dinâmico na programação do microfone direcional adaptativo automaticamente ativado. Com os relação aos algorítmos do AASI, optou-se por manter desligados o sound cleaner, o silenciador de ruído e o supressor de ruído de vento.

Os ajustes pertinentes à pesquisa foram feitos no AASI por meio dos programas NoahAud3 (NOAH System) e Beltone Solus versão 2.7.

Para a aplicação do Teste de Reconhecimento de Sentenças no Ruído, inicialmente, foram apresentadas 10 sentenças a $0^{\circ}$ azimute sem a presença do ruído e outras 10 sentenças apresentadas nesse mesmo ângulo na presença de ruído a $180^{\circ}$ azimute, para a familiarização do indivíduo com o teste. Nesse momento os indivíduos usavam os aparelhos com microfones onidirecionais. Essas medidas não foram consideradas na análise dos resultados.

Posteriormente, os três tipos de microfone foram testados em três situações de escuta distintas: fala apresentada num ângulo de $0^{\circ}$ azimute e ruído apresentado a $180^{\circ}$ azimute (fala na frente e ruído atrás); fala apresentada a $90^{\circ}$ azimute e ruído a $270^{\circ}$ azimute (fala à direita e ruído à esquerda); fala a $270^{\circ}$ azimute e ruído a $90^{\circ}$ azimute (fala à esquerda e ruído à direita). Para isso foram utilizadas as listas 1B, 2B, 3B, 4B, 5B, 6B e 7B do Compact Disc (CD).

As medidas foram obtidas em cabina tratada acusticamente, utilizando-se o audiômetro digital de dois canais da marca Interacoustics, modelo AC-33. As sentenças e o ruído foram apresentados por meio de um CD Player digital da marca Philips, modelo Jogproof, acoplado ao audiômetro acima descrito.

Considerando a necessidade de manter sempre as mesmas condições acústicas do ambiente durante toda a pesquisa as medidas em campo livre foram monitoradas pela examinadora com o auxílio de um Medidor de Nível de Pressão Sonora Digital da marca Realistic, para cada paciente avaliado.

Para estabelecer os parâmetros da calibração do canal das sentenças foi utilizado o tom puro presente na faixa 1 do CD. Para o ruído a calibração foi realizada utilizando o próprio ruído como referência, presente na faixa 18 . A saída de cada canal foi calibrada utilizando o VU-meter do audiômetro, ambos colocados no nível zero.

Para determinar o Limiar de Reconhecimento de Sentenças no Ruído (LRSR) foi utilizada a estratégia sequencial ou adaptaiva. Assim, foi apresentado o estímulo de fala em uma determinada relação $S / R$. Se o indivíduo fosse capaz de reconhecer corretamente a sentença a intensidade do estímulo era diminuída em intervalos pré-estabelecidos, caso contrário a intensidade era aumentada ${ }^{\text {. }}$.

Foram utilizados intervalos de $4 \mathrm{~dB}$ até a primeira mudança no tipo de resposta e posteriores intervalos de $2 \mathrm{~dB}$ até o final da lista. Uma resposta só foi considerada correta quando o indivíduo repetiu, sem erros ou omissões, a sentença apresentada.

Para a obtenção do valor da relação S/R o nível sonoro do ruído apresentado foi subtraído do nível sonoro em que foi obtido o LRSR. O nível sonoro do ruído foi mantido em $65 \mathrm{~dB}$ durante todas as medidas .

O presente estudo de caso foi aprovado pelo Comitê de Ética em Pesquisa da Associação Cultural e Científica Virvi Ramos, sob o protocolo de número 047/10. Todos os sujeitos assinaram o Termo de Consentimento Livre e Esclarecido (TCLE).

Considerando o tamanho da amostra, optou-se por elaborar o presente estudo de caso apresentando uma análise descritiva, a partir de medidas de tendência central obtidas para a relação $S / R$, com cada microfone avaliado em cada uma das situação de escuta investigadas.

\section{RESULTADOS}

A relação S/R consiste na diferença entre o nível sonoro do sinal de fala e o nível sonoro de um som competitivo. Quanto menor essa relação mais difícil é a situação de escuta. Assim, quanto menor o resultado da relação $S / R$ numa situação de teste, melhor é a habilidade do indivíduo.

No teste com o microfone omnidirecional, que capta o som proveniente de qualquer direção da mesma forma, referente à situação de escuta na qual o sinal de fala foi apresentado num ângulo de $0^{\circ}$ azimute e o ruído a $180^{\circ}$ azimute (fala na frente e ruído atrás), a média encontrada para a relação sinal/ruído (S/R) foi de 6,6 dB (Tabela 1). 
Tabela 1 - Medidas descritivas obtidas para a relação sinal/ruído em função dos microfones testados e da situação de apresentação dos estímulos

\begin{tabular}{lccccc}
\hline Variável & $\mathbf{n}$ & Mínimo & Máximo & Média & Desvio-padrão \\
$\begin{array}{l}\text { S/R omnidirecional fala na frente e } \\
\text { ruído atrás }\end{array}$ & 4 & 1,0 & 13,7 & 6,6 & 6,3 \\
$\begin{array}{l}\text { S/R omnidirecional fala à direita e } \\
\text { ruído à esquerda }\end{array}$ & 4 & $-8,7$ & 14,8 & 1,8 & 10,3 \\
$\begin{array}{l}\text { S/R omnidirecional fala à esquerda e } \\
\text { ruído à direita }\end{array}$ & 4 & $-4,5$ & 11,9 & 3,6 & 6,7 \\
$\begin{array}{l}\text { S/R direcional fixo fala na frente e } \\
\text { ruído atrás }\end{array}$ & 4 & $-2,0$ & 5,5 & 0,6 & 3,4 \\
$\begin{array}{l}\text { S/R direcional fixo fala à direita e } \\
\text { ruído à esquerda }\end{array}$ & 4 & $-8,7$ & 2,0 & $-3,5$ & 4,5 \\
$\begin{array}{l}\text { S/R direcional fixo fala à esquerda e } \\
\text { ruído à direita. }\end{array}$ & 4 & $-3,3$ & 4,2 & $-0,3$ & 3,6 \\
$\begin{array}{l}\text { S/R direcional adaptativo } \\
\text { automaticamente ativado fala na } \\
\text { frente e ruído atrás }\end{array}$ & 4 & $-6,1$ & 1,7 & $-1,5$ & 3,3 \\
$\begin{array}{l}\text { S/R direcional adaptativo } \\
\text { automaticamente ativado fala à direita } \\
\text { e ruído à esquerda }\end{array}$ & 4 & $-7,3$ & 4,6 & $-0,6$ & 5,0 \\
$\begin{array}{l}\text { S/R direcional adaptativo } \\
\text { automaticamente ativado fala à } \\
\text { esquerda e ruído à direita }\end{array}$ & 4 & $-11,3$ & $-2,5$ & $-6,9$ & 3,7 \\
\hline
\end{tabular}

Legenda: S/R: sinal/ruído

Na situação de escuta na qual o sinal de fala foi apresentado a $90^{\circ}$ azimute e o ruído a $270^{\circ}$ azimute (fala à direita e ruído à esquerda), a média da relação S/R encontrada foi de 1,8 dB (Tabela 1).

Ainda se tratando do microfone omnidirecional, na situação de escuta na qual o sinal de fala foi apresentado num ângulo de $270^{\circ}$ azimute e o ruído a um ângulo de $90^{\circ}$ (fala à esquerda e ruído à direita), foi encontrada uma média de 3,6 dB para a relação S/R (Tabela 1).

Portanto, ao testar-se nos quatro sujeitos o microfone omnidirecional, verificou-se que a melhor relação S/R obtida foi para a situação na qual a fala foi apresentada à direita e o ruído à esquerda.

Na mensuração utilizando o microfone direcional fixo, o qual capta melhor os sons vindos da frente e não modifica o padrão de resposta polar, no que se refere à situação de escuta na qual o sinal de fala foi apresentado num ângulo de $0^{\circ}$ azimute e o ruído a $180^{\circ}$ azimute (fala na frente e ruído atrás), a média da relação $S / R$ foi de 0,6 dB (Tabela 1).

Com esse mesmo microfone, na situação na qual o sinal de fala foi apresentado a $90^{\circ}$ azimute e o ruído a $270^{\circ}$ azimute (fala à direita e ruído à esquerda), a média encontrada para relação $S / R$ foi de $-3,5 \mathrm{~dB}$ (Tabela 1)
Ainda se tratando do microfone direcional fixo, na situação de escuta na qual o sinal de fala foi apresentado num ângulo de $270^{\circ}$ e o ruído a um ângulo de $90^{\circ}$ (fala à esquerda e ruído à direita), encontrou-se a média de -0,3 dB (Tabela 1).

Assim, na mensuração dos quatro sujeitos com o microfone direcional fixo, a melhor média para a relação $S / R$ também foi encontrada na situação na qual a fala foi apresentada à direita e o ruído à esquerda.

Na mensuração em que foram utilizados microfones direcionais adaptativos automaticamente ativados, os quais identificam o sinal de fala e mudam o padrão polar para que esse seja mais amplificado em relação ao ruído, na situação na qual o sinal de fala foi apresentado a $0^{\circ}$ azimute e o ruído incidiu num ângulo de $180^{\circ}$ (fala na frente e ruído atrás), a média encontrada para a relação $S / R$ foi de $-1,5 \mathrm{~dB}$ (Tabela 1).

Utilizando-se os mesmos microfones, na situação na qual o sinal de fala foi apresentado num ângulo de $90^{\circ}$ e o ruído num ângulo de $270^{\circ}$ (fala à direita e ruído à esquerda), a média da relação $S / R$ foi de $-0,6 \mathrm{~dB}$ (Tabela 1).

Já na avaliação desses microfones na situação de escuta na qual apresentou-se o sinal de fala a $270^{\circ}$ azimute e o ruído a $90^{\circ}$ azimute (fala à 
esquerda e ruído à direita), encontrou-se a média de $-6,9 \mathrm{~dB}$ (Tabela 1).

Em relação ao microfone direcional adaptativo automaticamente ativado a melhor média para a relação $S / R$ foi encontrada na situação na qual a fala incidiu à esquerda e ruído à direita.

Considerando os três microfones e as três situações de escuta testadas, a melhor relação sinal ruído encontrada foi de $-6,9 \mathrm{~dB}$ para a situação na qual a fala foi apresentada à esquerda e o ruído à direita com o microfone direcional adaptativo automaticamente ativado.

A média, encontrada para a relação $S / R$, considerando as três situações de apresentação dos estímulos, para o microfone ominidirecional, foi de 4 $\mathrm{dB}$. Para o microfone direcional fixo foi de $-1,06 \mathrm{~dB}$. Já para o microfone direcional adaptativo automaticamente ativado, a média encontrada foi de $-3 \mathrm{~dB}$.

Além dos aspectos apresentados acima, investigou-se também, por meio de uma análise qualitativa, a percepção dos sujeitos com relação ao seu desempenho comunicativo em ambientes silenciosos e ruidosos e sua avaliação subjetiva do desempenho dos diferentes microfones testados na pesquisa.

Ao serem investigadas as principais restrições comunicativas apresentadas pelos quatro sujeitos, verificou-se que apenas um sujeito referiu dificuldade para compreender a fala em ambiente silencioso, enquanto três sujeitos referiram dificuldade para compreender a fala em ambiente ruidoso.

$\mathrm{Na}$ questão na qual os sujeitos atribuíram uma nota de 1 a 10 para os microfones testados, considerando a facilidade para compreender a fala com cada uma deles, onde um correspondia a pouca facilidade e 10 a grande facilidade, a média encontrada para os microfones omnidirecionais foi 7,0 pontos $\pm 1,4$. Para o microfone direcional fixo a média foi de 7,3 pontos $\pm 2,1$. Para os microfones direcionais automáticos a média da nota dada foi 6,8 pontos $\pm 2,5$.

Os sujeitos responderam também em qual das situações de escuta houve melhor compreensão de fala, para cada um dos microfones. Para o microfone omnidirecional três sujeitos referiram que a situação na qual a fala é apresentada na frente e o ruído atrás é a mais fácil para compreender a fala e um sujeito não soube apontar.

No que diz respeito ao microfone direcional fixo, os quatro sujeitos referiram a situação na qual a fala é apresentada na frente e o ruído atrás como a que acreditavam terem obtido melhores resultados.

Para o microfone direcional adaptativo automaticamente ativado, três sujeitos apontaram a situação na qual a fala é apresentada na frente e o ruído atrás para essa pergunta e um deles apontou a situação na qual a fala foi apresentada a sua direita e o ruído à esquerda.

\section{DISCUSSÃO}

Em função do tamanho da amostra, o presente artigo refere-se a um estudo de quatro casos. Assim, entende-se que a variabilidade das respostas, o fator idade e os aspectos cognitivos, de memória de curto prazo, de atenção e de motivação para a realização do teste são fatores que devem ser considerados na interpretação dos resultados obtidos.

Em estudo que avaliou 14 indivíduos, usuários de AASI retroauricular monoaural ou binaural, com microfone omnidirecional $(n=9)$ e usuários de AASI retroauricular com microfone direcional $(n=5)$, que foram submetidos ao mesmo teste e tiveram os resultados da relação $S / R$ analisados, os pesquisadores concluíram que a direcionalidade favoreceu o reconhecimento de fala no ruído, porém não encontraram diferença estatística significante ${ }^{8}$.

As médias das relações $S / R$ encontradas no presente estudo mostraram resultados semeIhantes. O desempenho dos indivíduos utilizando os microfones direcionais foi melhor do que quando os mesmos utilizavam microfones omnidirecionais.

No estudo comparativo que investigou o desempenho de 17 indivíduos em testes de fala no silêncio e testes de fala no ruído para três diferentes tipos de microfones: microfone omnidirecional, microfone direcional fixo hipercardióide e microfone totalmente adaptativo ou automático, os resultados obtidos mostraram que, no silêncio, o desempenho dos indivíduos utilizando os microfones direcionais totalmente adaptativos foi semelhante ao dos microfones omnidirecionais. Os autores referiram que o microfone direcional fixo e o microfone totalmente adaptativo apresentaram resultados similares nas medidas envolvendo fala e ruído ${ }^{9}$.

Pode ser observada semelhança entre 0 presente estudo e o estudo supracitado no que diz respeito aos resultados obtidos com os microfones direcionais fixos e os microfones direcionais adaptativos automaticamente ativados nas mensurações envolvendo fala e ruído. Em ambos estudos os valores encontrados para os microfones com tecnologia direcional, seja ela fixa ou adaptativa, são semelhantes e se afastam dos valores encontrados com os microfones omnidirecionais.

Em estudo envolvendo 20 sujeitos, portadores de perda auditiva neurossensorial, já usuários de AASI, aplicou-se o Hearing in Noise Test com os indivíduos sem aparelho, com os próprios apareIhos, com o aparelho testado no modo omnidirecional e com o mesmo aparelho no modo direcional adaptativo automaticamente ativado. Constataram 
que o microfone direcional adaptativo automaticamente ativado proporcionou melhora na relação $\mathrm{S} / \mathrm{R}$ apresentada pelos indivíduos ${ }^{10}$. Os resultados desse estudo corroboram com os achados descritos acima, pois também pode-se perceber melhora na relação S/R com os microfones direcionais automaticamente ativados em relação aos demais.

Em estudo que teve por objetivo principal investigar se a relação $S / R$, medida em testes de inteligibilidade de fala no ruído, pode ser utilizada como uma medida representativa da distância necessária entre falante e ouvinte para fins de cálculos nos algorítmos de direcionalidade automática baseados na análise de diferentes ambientes ou cenas, os autores investigaram também a vantagem do microfone direcional para variadas relações $S / R$, utilizando a lista de sentenças IIEE/Harvard, apresentada na frente do indivíduo e o ruído apresentado atrás do mesmo. Constataram que houve vantagem do microfone direcional em relação ao microfone omnidirecional, proporcionando uma significante melhora na compreensão de fala. Entretanto houve grande variabilidade individual, pois ao analisar as respostas de cada sujeito, individualmente, verificaram que em algumas situações o desempenho foi melhor com o microfone omnidirecional ${ }^{11}$.

No presente estudo, os resultados quantitativos apontaram uma vantagem em dB na média encontrada para os microfones adaptativos automaticamente ativados.

Contudo, ao ser analisado o questionário qualitativo, os indivíduos, de um modo geral, deram notas maiores para os microfones omnidirecionais.

Em outra pesquisa, que investigou o efeito da assimetria de microfones direcionais na compreensão da fala no ruído por meio do Hearing in Noise Test e do Acceptable Noise Level, os autores avaliaram sujeitos utilizando microfones omnidirecionais, direcionais e direcional e omnidirecional alternados entre as orelhas. Para tal investigação, utilizaram o ângulo de apresentação do sinal de fala foi $0^{\circ}$ azimute e o ruído $180^{\circ}$ azimute. Os resultados obtidos pelos autores demonstraram que a compreensão da fala no ruído foi melhor quando os indivíduos utilizavam microfones assimétricos do que os demais, sendo estatísticamente significante apenas em relação aos microfones omnidirecionais binaurais. Com relação ao nível de aceitação do ruído os melhores resultados foram obtidos para os microfones direcionais binaurais ${ }^{12}$.

Estudo comparou a relação S/R obtida no teste LRSR entre indivíduos normo-ouvintes e portadores de perda auditiva neurossensorial de grau leve a moderadamente severo. A média encontrada para indivíduos normo-ouvintes foi de $-7,57 \mathrm{~dB}$, enquanto que para os portadores de perda auditiva foi de
$-2,10 \mathrm{~dB}{ }^{7}$. Nesse estudo, foram obtidos valores maiores que $-2,10 \mathrm{~dB}$, mesmo com o uso de AASI.

$A$ melhor média da relação $S / R$ encontrada nesse estudo foi na mensuração com os microfones automáticos na situação na qual a fala foi apresentada à esquerda e o ruído à direita ( $\mathrm{S} / \mathrm{R}-6,9 \mathrm{~dB})$. Esse resultado se aproxima do encontrado para indivíduos normo-ouvintes no estudo citado acima. Entretanto, é possível que esta relação mais favorável esteja relacionada com o efeito de aprendizagem, já que essa foi a última situação medida nos quatro casos e que esse valor se distancia dos demais encontrados, até para os mesmos microfones.

Em outro estudo que avaliou, objetiva e subjetivamente, a inteligibilidade de fala no ruído de 18 usuários de AASI que dispunha de microfone direcional totalmente adaptativo e algorítmo de redução de ruído, os autores verificaram que ambos os recursos haviam trazido benefício para o reconhecimento de fala no ruído dos usuários. Os microfones direcionais, no entanto, foram os que apresentaram maior benefício, de acordo com o que foi relatado pelos indivíduos ${ }^{13}$.

Em estudo que comparou a relação S/R obtida para indivíduos normo-ouvintes com o ruído incidindo em diferentes ângulos $\left(0^{\circ} / 0^{\circ}, 0^{\circ} / 90^{\circ}, 0^{\circ} / 180^{\circ}\right.$ e $0^{\circ} / 270^{\circ}$ azimute), os autores constataram que, das situações avaliadas, aquela na qual a fala e o ruído provinham do mesmo ângulo revelou-se como a mais difícil. A situação na qual o ruído vinha de trás e a fala da frente foi a segunda em termos de dificuldade ${ }^{14}$.

A média da relação $S / R$ encontrada para a situação de teste na qual a fala advinha da frente e o ruído de trás $\left(0^{\circ} / 180^{\circ}\right)$ no estudo supracitado, foi de $-9,751 \mathrm{~dB}$. No presente estudo, a média da relação $S / R$ encontrada para a mesma situação foi de $6,6 \mathrm{~dB}$ para os microfones omnidirecionais, 0,6 $\mathrm{dB}$ para os microfones direcionais e $-1,5 \mathrm{~dB}$ para os microfones direcionais automáticos. Essa diferença pode estar relacionada ao fato de que os indivíduos que participaram dessa pesquisa eram portadores de perda auditiva neurossensorial e nunca haviam usado AASI anteriormente.

Já a situação mais referida como mais fácil no presente estudo foi $\left(0^{\circ} / 180^{\circ}\right)$. Apenas um sujeito, em uma situação de escuta específica, referiu que acreditava ser mais fácil compreender quando a fala foi apresentada à direita e o ruído à esquerda $\left(90^{\circ} / 270^{\circ}\right)$.

Percebe-se que a escolha dos microfones é apenas um dos aspectos quando se tem por objetivo melhorar o reconhecimento de fala no ruído em usuários de AASI. Acredita-se que o treinamento auditivo, no período de pós adaptação, contribua 
para o aprimoramento dessa habilidade. Esse não é, de forma alguma, substituído por modificações nos algorítmos dos aparelhos.

\section{CONCLUSÃO}

A média das relações sinal/ruído variou de 6,6 $\mathrm{dB}$ a $-6,9 \mathrm{~dB}$. O microfone que apresentou melhor média para a relação sinal/ruído, considerando as três situações de apresentação dos estímulos, foi o direcional adaptativo automaticamente ativado. Entretanto, por se tratar de uma amostra pequena, houve grande variabilidade individual. Em virtude de se tratar de um estudo envolvendo somente 04 indivíduos, com quais se testou um único tipo de equipamento, e de que as diferenças de tecnologia entre os microfones do aparelho testado são, provavelmente, mínimas, novos estudos devem ser realizados a fim de que se tenha subsídios científicos para a indicação dos microfones mais apropriados para minimizar as dificuldades de compreensão de fala no ruído. O Fonoaudiólogo, que atua na área de prótese auditiva, deve compreender e dominar plenamente os recursos tecnológicos disponíveis no mercado e estar sempre atualizado para que possa tirar o máximo proveito possível desses no auxilio aos pacientes.

\section{AGRADECIMENTOS}

À Audibel, aparelhos auditivos, pela concessão dos Aparelhos de Amplificação Sonora Individual Reach, RCH62, Beltone. Às fonoaudiólogas Circe Nardon Carvalho de Miranda e Sandra Regina de Siqueira Braga pelo apoio durante a realização dessa pesquisa.

\begin{abstract}
The difficulty of understanding speech with background noise is perceived as one of the main disabilities of the hearing aids users. The purpose of this study was to compare the hearing performance of subjects with sensorioneural, bilateral, light to moderate degree hearing loss with the microphones omnidirectional, fixed directional mode and automatically activated adaptive directional mode, activated through the signal/noise ratio $(S / R)$ in which the Sentence Recognition Threshold in Noise are obtained. It was used the hearing aid Reach, $\mathrm{RCH} 62$, Beltone in omnidirectional, fixed directional and automatically activated adaptive microphone modes. The following presentations of acoustic stimulus had been tested: speech $0^{\circ}$ azimuth and noise $180^{\circ}$ azimuth $\left(0^{\circ} / 180^{\circ}\right)$, speech $90^{\circ}$ azimuth and noise $270^{\circ}$ azimuth $\left(90^{\circ} / 270^{\circ}\right)$ and speech $270^{\circ}$ azimuth and noise $90^{\circ}$ azimuth $\left(270^{\circ} / 90^{\circ}\right)$. The average signal to noise ratio ranged from $6,6 \mathrm{~dB}$ to $-6,9 \mathrm{~dB}$. The microphone that had the best average for signal to noise ratio, considering the three conditions of stimulus presentation, was the automatically activated adaptive. However, because it is a small sample, there was great individual variability. Further studies should be performed to obtain scientific support for the most appropriate selection of microphones.
\end{abstract}

KEYWORDS: Hearing Aid; Hearing Loss; Speech Intelligibility; Noise

\section{REFERÊNCIAS}

1. Gama MR. Percepção da Fala: uma proposta de avaliação qualitativa. São Paulo: Pancast; 1994.

2. lório MCM, Menegotto IH. Próteses Auditivas. In: Filho OL[ed]. Tratado de Fonoaudiologia. Ribeirão Preto: Tecmedd; 2005.p.449-69.

3. Fabry DA. Adaptive Directional Microphone Technology and Hearing Aids: Theoretical and Clinical Implications. Hearing Review. 2005 Disponível em: http://www.hearingreview.com/ issues/articles/2005-04_02.asp Acesso em: 18 de julho de 2011.

4. Ricketts T, Henry P, Gnewikow D. Full time directional versus user selectable microphone modes in hearing aids. Ear Hear. 2003;24(5):424-39. 5. Mills RJ, Martin D. Why Don't Directional Microphones Work Better? Hearing Review. 2007 Disponível em: http://www.hearingreview.com/ issues/articles/2007-09_04.asp Acesso em: 10 de agosto de 2011.

6. Frazza MM, Caovilla HH, Munhoz MSL, Silva MLG, Ganaça MM. Audiometria Tonal e Vocal. In: 
Munhoz MSL Caovilla HH, Silva MLG, Ganaça MM [ed]. Audiologia Clínica. São Paulo: Atheneu; 2003. p.56.

7. Henriques MO, Costa MJ. Reconhecimento de Sentenças no Ruído, em campo livre, em indivíduos com e sem perda auditiva. Rev. CEFAC. 2011. Disponível em: http://www.scielo.br/pdf/ rcefac/2011nahead/120-10.pdf Acesso em: 12 de agosto de 2011.

8. Quintino CA, Mondelli MFCG, Ferrari DV. Directivity and noise reduction in hearing aids: speech perception and benefit. Braz $\mathrm{J}$ Otorhinolaryngol. 2010;76(5):630-8.

9. Kuk F, Keenan D, Lau CC, Ludvigsen C. Performance of a fully adaptive directional microphone to signals presented from various azimuths. J Am Acad Audiol. 2005;6(6):333-47.

10. Valente $M$, Mispagel KM. Performance of an Automatic Adaptive Dual-Microphone ITC Digital
Hearing Aid. Hearing Review. 2004; Disponível em: http://www.hearingreview.com/issues/articles/ 2004-02_05.asp Acesso em: 15 de julho de 2011.

11. Walden BE, Surr RK, Grant KW, Van Summers W, Cord MT, Dyrlund O. Effect of signal-to-noise ratio on directional microphone benefit and preference. J Am Acad Audiol. 2005;16(9):662-76.

12. Kim JS, Bryan MF. The effects of asymmetric directional microphone fittings on acceptance of background noise. Int J Audiol. 2011;50(5):290-6.

13. Peeters H, Kuk F, Lau CC, Keenan D. Subjective and objective evaluation of noise management algorithms. J Am Acad Audiol. 2009; 20(2):89-98.

14. Henriques MO, Costa MJ. Limiares de reconhecimento de sentenças em indivíduos normo-ouvintes na presença de ruído incidente de diferentes ângulos. Rev Soc Bras Fonoaudiol. 2011;16(1):54-8.
http://dx.doi.org/10.1590/S1516-18462012005000097

Recebido em: 04/11/2011

Aceito em: 04/02/1012

Endereço para correspondência:

Mariane Turella Mazzochi

VRS 829, n. 4120, Fazenda Souza

Caxias do Sul - RS - Brasil

CEP: 95125-000

E-mail: mah_mazzochi@hotmail.com 